DE92 004369

\title{
Relativistic Meson-Exchange Currents in the Dip Region
}

\author{
M.J. Dekker and P.J. Brussaard \\ R.J. Van de Graaff Laboratory, University of Utrecht \\ P.O. Box 80.000, 3508 TA Utrecht, The Netherlands
}

\author{
J.A. Tjon \\ Institute for Nuclear Theory, University of Washington \\ HN-12, Seattle, Washington 98195, USA \\ and Institute for Theoretical Physics, University of Utrecht \\ 3508 TA Utrecht, The Netherlands
}

\section{PREPARED FOR THE U.S. DEPARTMENT OF ENERGY}

This report was prepared as an account of work sponsored by the United States Government. Neither the United States nor the United States Department of Energy, nor any of their employees, nor any of their contractors, subcontractors, or their employees, makes any warranty, express or implied, or assumes any legal liability or responsibility for the product or process disclosed, or represents that its use would not infringe privately-owned rights.

By acceptance of this article, the publisher and/or recipient acknowledges the U.S. Government's right to retain a nonexclusive, royaity-free license in and to any copyright covering this paper.

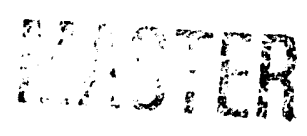




\title{
Relativistic Meson-Exchange Currents in the Dip Region
}

\author{
M.J. Dekker and P.J. Brussaard \\ R.J Van de Graaff laboratory, University of Utrecht \\ P.O Box 80.000, 3508 TA Utrecht, The Netherlands.
}

J.A. Tjon

Institute for Nuclear Theory, University of Washington

Seattle, WA 98195, USA and Institute for Theoretical Physics

University of Utrecht, 3508 IA Utrecht, The Netherlands.

\begin{abstract}
We show that a relativistic treatment of meson exchange currents for inclusive electron scattering off a nucleus treated as a free Fermi gas leads to a strong enhancement of the transverse response function in the dip region in the two-particle knockout channel, mainly through the intermediate $\Delta$ (1232)-excitation. This suggests that the missing strength in the dip region can, at least partially, be accounted for by a relativistic treatment of the $\Delta(1232)$-excitation and its coupling to the two-nucleon system. Recoil effects are found to be sizable over the whole range of energy transfer including the quasi-elastic peak.
\end{abstract}


Considerabie effort has been put into the theoretical description of electronnucleus scattering data [1]. For different kinematical domains (i.e. differing in energy and momentum transfer to the nucleus by the scattered electron) different dynamical models have been studied. With increasing momentum transfer it is an interesting question if and where in these models special relativity plays a role. Due to gauge invariance the choice of the electromagnetic operator is not independent from the strong interaction between the hadrons. For the two-nucleon system[2] a consistent relativistic treatment has recently been carried out. In particular, it was shown that the use of a fully relativistic electromagnetic operator, including also the recoil effects, may lead to very different results as compared to a static treatment. For many-body systems an adequate relativistic description of the strongly interacting system is at present not available. Here one has to resort to simple models and hope to find qualitative trends which can be compared with experiment.

In this letter we are interested in the meson exchange current (MEC) contributions in the kinematical domain of the quasi-elastic (QE) peak and the $\Delta(1232)$-resonance region with emphasis on the dip region, the region of energy transfer between the $\mathrm{QE}$ peak and the $\Delta(1232)$-resonance. Both the $\mathrm{QE}$ peak and the $\Delta(1232)$-resonarice are usually described by means of single-particle models, where essentially all energy and momentum are being transferred to a single nucleon. These models fail to describe the observed nuclear response in the dip region. Moreover, when a separation of the total nuclear response into its transverse and longitudinal parts is made, refinements of these models fail to improve the description of these separate responses simultaneously. It has been suggested that for thcse kinematics two- and more-particle processes might be important. Natural candidates for these processes besides finalstate interaction effects, are MEC contributions. To estimate their importance, Van Orden, Donnelly, deForest and Hermans [3,4] calculated MEC contributions to the transverse nuclear response function in a Fermi gas model. In these calculations the usual non-relativistic reduction of the MEC was considered. The importance of the non-relativistic MEC is relatively small in both the $\mathrm{QE}$ peak and the dip region. The 
main purpose of this work is to study these contributions using the fully relativistic MEC operator. As in the case of the deuteron we find that the resulting operators may yield quantitatively very different results from the static-limit approximation, which shows that at high momentum transfer recoil contributions are important.

The differential cross-section for inclusive electron scattering in the one-photon exchange approximation is given by [5]

$$
\frac{\mathrm{d}^{2} \sigma}{\mathrm{d} \Omega \mathrm{d} E}=\sigma_{\mathrm{M}}\left(\left(\frac{q^{2}}{\vec{q}^{2}}\right)^{2} R_{\mathrm{L}}(\vec{q}, \omega)+\left(\frac{-q^{2}}{2 \vec{q}^{2}}+\tan ^{2} \frac{\theta}{2}\right) R_{\mathrm{T}}(\vec{q}, \omega)\right),
$$

where $\sigma_{M}$ is the Mott cross-section for scattering off a point proton. We use Bjorken and Drell conventions [6]. By means of a Roserbluth separation one can divide the measured total cross-section into its longitudinal $\left(R_{\mathrm{L}}\right)$ and transverse $\left(R_{\mathrm{T}}\right)$ parts $[7,8]$. The generic feature of these two response fuctiors for differing mass numbers $A$ is that the longitudinal part drops to zero for large energy transfer $\omega$, while the transverse part only slightly decreases for high energy transfer, thus filling the dip between the $\mathrm{QE}$ peak and the $\Delta(1232)$ resonance. The transverse response function $R_{\mathrm{T}}$ is determined by the nuclear current distribution

$$
R_{\mathrm{T}}=-\left(g_{i j}+\frac{q_{i} q_{j}}{\vec{q}^{2}}\right) W^{i j}
$$

with

$$
W^{\mu \nu}=(2 \pi)^{3} V \overline{\sum_{i}} \sum_{\mathbf{f}}<\mathrm{f}\left|\hat{J}^{\mu}\right| \mathrm{i}>^{*}<\mathrm{f}\left|\hat{J}^{\nu}\right| \mathrm{i}>\delta^{4}\left(p_{\mathrm{i}}-p_{\mathrm{f}}+q\right) .
$$

Here $V$ is the normalization volume for the nuclear wavefunctions and $\hat{J}$ is the full nuclear current operator.

In the very simple model we use, the nucleus is described by a free Fermi gas in which all nucleons are on the mass shell. Nucleons are thus described by free Dirac spinors with mass $M=939 \mathrm{MeV}$. As initial state we take the filled Fermi sphere. The final state that we study is a state in which two nucleons are excited above the Fermi sphere, leaving two holes. Without final-state interactions, this state can only be reached by a two-body current. It is easy to see that in this model this channel adds incoherently to channels exciting only one particle above the Fermi sphere. 
When both initial and final states are correctly anti-symmetrized, one obtains two different contributions to $R_{\mathrm{T}}$, a direct part and a Pauli-exchange part. The Pauliexchange term vanishes for the pion current, due to isospin considerations, while it is expected to be relatively small for the $\Delta$-current in the considered kinematical region [4]. We therefore neglected it in this study. The final states are characterized by the allowed momenta, spins and isospins of the two excited particles and the remaining holes, which should all be summed over. The momentum sums can be changed into integrals where the mass shell conditions leave us with three-dimensional integrations. Finally the sum over spins can be transformed into a trace in spinor space by using $\sum_{s} U(p, s) \bar{U}(p, s)=(\gamma \cdot p+M) / 2 M$. As a result, we get for the twc-body current contribution to the transverse response, through the $2 \mathrm{p}-2 \mathrm{~h}$ channel,

$$
\begin{aligned}
W_{(2,2)}^{\mu \nu}= & \frac{1}{4}(2 \pi)^{3} V \sum_{\text {isospin }} \int \frac{\mathrm{d}^{3} p_{1} \mathrm{~d}^{3} p_{2} \mathrm{~d}^{3} p_{1}^{\prime} \mathrm{d}^{3} p_{2}^{\prime}}{(2 \pi)^{12}} \frac{M^{4}}{(2: M)^{4} E_{p_{1}} E_{p_{2}} E_{p_{1}^{\prime}} E_{p_{2}^{\prime}}} \times \\
& 2 \operatorname{Tr}_{1,2}\left(\left(\gamma \cdot p_{1}^{\prime}+M\right)\left(\gamma \cdot p_{2}^{\prime}+M\right) \hat{J}_{(2)}^{\nu}\left(\gamma \cdot p_{1}+M\right)\left(\gamma \cdot p_{2}+M\right) \gamma_{0} \hat{J}_{(2)}^{\mu^{\dagger}} \gamma_{0}\right) \times \\
& \theta\left(k_{\mathrm{F}}-p_{1}\right) \theta\left(k_{\mathrm{F}}-p_{2}\right) \theta\left(p_{1}^{\prime}-k_{\mathrm{F}}\right) \theta\left(p_{2}^{\prime}-k_{\mathrm{F}}\right) \delta^{4}\left(p_{1}+p_{2}+q-p_{1}^{\prime}-p_{2}^{\prime}\right)
\end{aligned}
$$

The factor $1 / 4$ is a statistical factor accounting for the fact that we sum over pairs of indistinguishable particles and holes. The factor 2 stems from the equality of the product of the direct terms and the product of the two exchange terms after interchange of indices. The mass shell energy is given by: $E_{p}=\sqrt{\vec{p}^{2}+M^{2}}$.

By choosing a set of Feynman cliagrams, $\hat{J}_{(2)}^{\mu}$ can now be constructed from an appropriate Lagrangian. For the sake of comparison we use the same Lagrangian as in Ref. [4]. This Lagrangian, originating from Peccei [10], is constructed by demanding chiral symmetry. It therefore possesses a pseudo-vector $\pi \mathrm{NN}$ coupling. The vertices containing the spin-3/2 Rarita-Schwinger $\Delta(1232)$-field are constrained by the requirement that this field is not allowed to couple to its spin-1/2 content[11]. The set of diagrams used to construct the two-body current is given in Fig. 1. Both strong and electromagnetic form factors are included in the calculations. For the electromagnetic form factors we take the Höhler [12] parametrization both for the $\gamma \Delta \mathrm{N}$ and $\gamma \mathrm{NN}$ vertices. For the strong form factors we take a dipole form with cutoffs 
$\Lambda_{\mathrm{N}}^{2}=1.5 M_{\mathrm{N}}^{2}$ for the $\pi N N$ and $\Lambda_{\Delta}^{2}=1.3 M_{\mathrm{N}}^{2}$ for the $\pi \Delta \mathrm{N}$ vertices respectively. In the Rarita-Schwinger propagator a complex $\Delta(1232)$-mass with an energy dependent width $\Gamma$ has been used, that is

$$
M_{\Delta} \rightarrow M_{\Delta}-i \Gamma\left(\sqrt{s_{\pi \mathrm{N}}}\right) / 2,
$$

where $s_{\pi \mathrm{N}}$ is the invariant mass square of the $\pi \mathrm{N}$ system. For the $\Delta(1232)$-width we take the Bransden-Moorhouse parametrization [13]. Below pion production threshold $\Gamma$ is taken to vanish. Current conservation is taken care of by using the method of Gross and Riska [14]. We do not make a non-relativistic reduction of the current and stay in the momentum representation. Therefore their method to construct a gauge invariant current including form factors, simply using the freedom of adding transverse terms which are separately conserved, can be used. The current one gets this way from diagram (b) of Fig. 1 then turns out to be a direct covariant generalization of the static current given in [15], where counterterms have been added to restore gauge invariance. The actual algebraic expression resulting from the evaluation of the traces in equation (4) is obtained by using the algebraic manipulation program FORM [16].

For the comparison of our results with the results of [4] and [17] some care must be taken in the treatment of the delta propagator in the static-limit. To get this static-limit we may replace the full covariant propagator of the Rarita-Schwinger spin-3/2 field by its positive-energy part

$$
P_{+}^{\mu \nu}=\frac{\gamma \cdot \hat{p}+M_{\Delta}}{2 E_{p}\left(p_{0}-E_{p}\right)}\left(-g^{\mu \nu}+\frac{2}{3 M_{\Delta}^{2}} \hat{p}^{\mu} \hat{p}^{\nu}+\frac{1}{3 M_{\Delta}}\left(\hat{p}^{\nu} \gamma^{\mu}-\hat{p}^{\mu} \gamma^{\nu}\right)+\frac{1}{3} \gamma^{\mu} \gamma^{\nu}\right),
$$

where we denote $\hat{p}=\left(E_{p}, \vec{p}\right), E_{p}=+\sqrt{\vec{p}^{2}+M_{\Delta}^{2}}$ and $p_{0}=M$ in the static-limit. Because the propagator is contracted with a vertex $\mathcal{O}^{\nu \rho}$, which by construction obeys the condition $\gamma_{\nu} \mathcal{O}^{\nu \rho}=0$, we are effectively left with

$$
P_{+}^{\mu \nu}=\frac{\gamma \cdot \hat{p}+M_{\Delta}}{2 E_{p}\left(p_{0}-E_{p}\right)}\left(-g^{\mu \nu}+\frac{2}{3 M_{\Delta}^{2}} \hat{p}^{\mu} \hat{p}^{\nu}+\frac{1}{3 M_{\Delta}} \hat{p}^{\nu} \cdot \gamma^{\mu}\right)
$$

instead of

$$
P^{\mu \nu}=\frac{\gamma \cdot p+M_{\Delta}}{p^{2}-M_{\Delta}^{2}}\left(-g^{\mu \nu}\right)
$$


with $p_{0}=M$ and $p^{2}=M^{2}$ in the static-limit. In doing so we obtain for the nonrelativistic $\Delta(1232)$-exchange current operator of diagrams (d) to (f) of Fig. 1

$$
\begin{aligned}
& \bar{U}\left(p_{1}^{\prime} s_{1}^{\prime}, \rho_{1}^{\prime}\right) \bar{U}\left(p_{2}^{\prime} s_{2}^{\prime}, \rho_{2}^{\prime}\right) \hat{\vec{J}}_{\Delta} U\left(p_{1}, s_{1}, \rho_{1}\right) U\left(p_{2}, s_{2}, \rho_{2}\right) \stackrel{\text { def }}{=} \vec{J}_{\Delta}\left(p_{1}, p_{2}, p_{1}^{\prime}, p_{2}^{\prime}\right), \\
& \vec{J}_{\Delta}\left(p_{1}, p_{2}, p_{1}^{\prime}, p_{2}^{\prime}\right) \stackrel{\text { stat. Lim. }}{=} \frac{k^{*} h f}{2 M m_{\pi}^{2}}\left(\frac{2 M}{V}\right)^{2} \times \\
& \chi_{a_{1}^{\prime}}^{\dagger} \chi_{s_{2}^{\prime}}^{\dagger} \chi_{\rho_{1}^{\prime}}^{\dagger} \chi_{\rho_{2}^{\prime}}^{\dagger}\left[\left(i 2 A\left(\vec{q} \times \vec{k}_{2}\right) \frac{\vec{\sigma}_{2} \cdot \vec{k}_{2}}{\vec{k}_{2}^{2}+m_{\pi}^{2}} \frac{2}{3} \tau_{1}^{3}+(1 \leftrightarrow 2)\right)-\right. \\
&\left.\left(2 B \frac{\vec{\sigma}_{2} \cdot \vec{k}_{2}}{\vec{k}_{2}^{2}+m_{\pi}^{2}} \vec{q} \times\left(\vec{k}_{2} \times \vec{\sigma}_{1}\right) \frac{i}{3}\left(\vec{\tau}_{1} \times \vec{\tau}_{2}\right)^{3}-(1 \leftrightarrow 2)\right)\right] \chi_{s_{1}} \chi_{s_{2}} \chi_{\rho_{1}} \chi_{\rho_{2}}
\end{aligned}
$$

with

$$
A=\frac{8}{3} \frac{1}{M-M_{\Delta}} \text { and } B=\frac{4}{3} \frac{1}{M-M_{\Delta}} .
$$

The values of the coupling-constants $k^{*}, h$ and $f$ will be given below. The result is different from that obtained in [4], where some terms of leading order have been neglected. Numerically the difference between the two operators is found to be small.

By makirg use of energy and momentum conservation and the rotational symmetry around the external vector $\vec{q}$, the twelve-dimensional integral in equation (4) can be reduced to seven dimensions. These integrals have been calculated by Monte Carlo integration. In [4] a method was developed to reduce the integral further to two dimensions, which is applicable to the static electromagnetic operator only. As a check for the numerics it was verified that in this case the same results as in $[4,17]$ were obtained both with seven-dimensional Monte Carlo integration and with the two-dimensional integration method.

The various coupling constants which have been used in the calculations are the same as in [4]. We have $f_{\pi N N}^{2} / 4 \pi=0.08$ and $f_{\pi N \Delta}^{2} / 4 \pi=(4 h)^{2} / 4 \pi=0.37$ en $f_{\gamma N \Delta}^{2} / 4 \pi=\left(\frac{m_{\pi} k^{*}}{2 M}\right)^{2} / 4 \pi=0.011$. In Figs. 2 and 3 we display the results of our calculation of the contribution of $W_{(2,2)}^{\mu \nu}$ to the transverse response for a fixed momentum transfer $|\vec{q}|=550 \mathrm{MeV} / c$ and two different values of the Fermi monentum $k_{\mathrm{F}}$ as a function of the energy transfer $\omega$. For comparison the static-limit results are 
also shown. Note the large difference between the two treatments for energy transfer above the QE peak. Since it is expected that in the $\omega$-region up to the delta peak, the $1 \mathrm{p}-1 \mathrm{~h}$ response from the direct single-nucleon knockout and the isobar production process is important, we have also determined these contributions in the same free Fermi gas retaining relativistic kinematics and using the isobar model[18, 9]. To calculate these contributions the same form factors and coupling constants as in our two-body current analysis have been used. As can be seen from the figures the total transverse response function, obtained by summing the above two $1 \mathrm{p}-1 \mathrm{~h}$ contributions and $W_{(2,2)}$, is found to be in reasonable agreement with the experimental data $[7,8]$.

Several points are worth mentioning about the various two-body current contributions. If only the MEC due to pions is considered, recoil effects are relatively small. There is an enhancement of the response function as compared to the static-limit results of about $7-15 \%$ for the contact current and $10-20 \%$ for the pion-in-flight current, if one keeps in both the full and the static calculation the normalization factor $M / E_{p}$. If one puts this factor equal to unity in the latter calculation, the difference between the two becomes small. However, inclusion of the $\Delta(1232)$-exchange current gives rise to a substantially larger response for energy transfer above the $Q E$ peak, which results in a better agreement with experiment. It reflects the resonant behaviour of the $\Delta(1232)$-excitation as a function of the energy transfer. This is properly accounted for in the complete electromagnetic nperator, whereas this is not the case in the static-limit. At high enough energy transfer the production of on-shell pions is possible, which is described here by the energy dependent $\Delta(1232)$-width. In the considered $\omega$-region this contribution is small however. The contribution of our $W_{(2,2)}^{00}$ to the longitudinal response is very small, as it is expected to be. For large $\omega$ it also shows the resonance behaviour, but the resulting response lies well within the experimental results. Our results depend quite strongly on the value of the Fermi momentum $k_{\mathrm{F}}$, as can be seen from a comparison between Figs. 2 and 3. Apart form a trivial scaling with the atomic mass number $A, k_{\mathrm{F}}$ is the only parameter that has 
been changed in the calculation leading to the results of Fig. 3 compared with those of Fig. 2. It is interesting to note that for larger Fermi momentum our calculated $2 \mathrm{p}-2 \mathrm{~h}$ response becomes relatively larger. This is in accord with the experimental observation that the dip becomes more filled for larger values of the mass number $A$, i.e. for increasing Fermi momentum. Although the agreement is gratifying for a set of reasonable cut-off masses in the strong form factors, it should be noted that the predictions are clearly sensitive to the choice of these cut-off masses.

In conclusion, we have found that our complete treatment of the electromagnetic MEC operator leads to substantially different results as compared to the usual static-limit treatment. In general the contributions are significantly larger in the dip region yielding a closer agreement with experiment. This is mainly due to a resonance behaviour in the $\triangle(1232)-\mathrm{MEC}$, which we treat without making any static approximation. This MEC can be viewed as a description of the coupling of the $\Delta(1232)$-resonance to two-nucleon final states. We wish to emphasize that our completely uncorrelated Fermi gas ground state wavefunction is clearly too simple. For a more complete analysis the final-state interaction effects have to be considered, together with other contributions like the Pauli-exchanged MEC and the pair term. It should be noted, that the latter would automatically be included in a relativistic treatment of the one-body current contributions and the final-state interaction effects and moreover there would also be an interference between one-body and two-body currents in the $2 \mathrm{p}-2 \mathrm{~h}$ channel. From our study we may conclude that for a reliable estimate of the contributions of the various two-body currents the full relativistic electromagnetic operators must be used. The present results are encouraging as the MEC contributions are significantly larger than was expected from a non-relativistic analysis. As a result, a sizable part of the response in the dif region may be indeed due to these MEC contributions.

\section{Acknowledgement}

This work was supported in part by the Stichting voor Fundamenteel Onderzoek 
der Materie (FOM), which is sponsored by the Nederlandse Organisatie voor Wetenschappelijk Onderzoek (NWO), and by the US Department of Energy. One of us

(M.J.D.) wants to thank J. Haveman for his computer guidance.

\section{References}

[1] S. Frullani and J. Mougey, Adv. Nucl. Phys. 141 (1984).

[2] E. Hummel and J.A. Tjon, Phys. Rev. Lett. 631788 (1989).

[3] W. Van Orden, T.W. Donnelly, T.deForest Jr. and W.C. Hermans, Phys. Lett. B76 393 (1978)

[4] W. Van Orden and T.W. Donnelly, Ann. Phys. (N.Y.) 131451 (1981).

[5] T.deForest and J.D. Walecka, Adv. in Phys. 151 (1966).

[6] J.D. Bjorken and S.D. Drell, Relativistic quantum fields, McGraw-Hill Book Compar., New York (1964).

[7] P. Barreau et al. , Nucl. Pys. A402 515 (1983).

[8] Z... Mezziani et al. , Phys. Rev. Lett. 541233 (1985).

[9] W.Van Orden, Ph.D Thesis, Stanford University (July 1978).

[10] R.D. Peccei, Phys. Rev. 1761812 (1968) ;181 1902 (1969).

[11] M. Veltman, in Weak Interactions and High-Energy Neutrino Physics, International School of Physics Enrico Fermi, Course XXXII, 1964 (ed. T.D. Lee), Academic Press, New York (1966).

[12] G. Höhler et al. ,Nucl. Phys. B114 505 (1976).

[13] B.H. Bransden and R.G. Moorhouse, The Pion-Nucleon System, Princeton University Press, Princeton (1973). 
[14] F. Gross and D.O. Riska, Phys. Rev. C36 1928 (1987) and private communication.

[15] J. -F. Mathiot, Phys. Rep. 17365 (1989).

[16] FORM, an algebraic manipulation program for large formulae, written by J. Vermaseren (NIKHEF-H, Amsterdam, the Netherlands).

[17] W.M. Alberico, M. Ericson and A. Molinari, Ann. Phys. (N.Y.) 154356 (1984).

[18] E.J. Moniz, Phys. Rev. 1841154 (1969). 


\section{Figure Captions.}

Fig. 1. Feynman diagrams used to construct the two-body current operator. The exchanged mesons are pions, the intermediate baryon is the $\Delta(1232)$-resonance.

Fig. 2. Different contributions to the transverse response function in a free Fermi gas model for a fixed momentum transfer of $550 \mathrm{MeV} / \mathrm{c}$ and Fermi momentum of $1.15 \mathrm{fm}^{-1}$. Dash-dotted curve: our relativistic $2 \mathrm{p}-2 \mathrm{~h}$ response, short-dashed curve: static-limit $2 \mathrm{p}-2 \mathrm{~h}$ response, dotted curve: $1 \mathrm{p}-1 \mathrm{~h}$ response in free Fermi gas model, long-dashed curve: resonant part of the $1 \mathrm{p}-1 \mathrm{~h}$ response in the isobar model, solid curve: sum of $1 \mathrm{p}-1 \mathrm{~h}$ and relativistic $2 \mathrm{p}-2 \mathrm{~h}$ responses. Triangles are data for ${ }^{12} \mathrm{C}$ taken from [7].

Fig. 3. Same as Fig. 2 but here the Fermi momentum is $1.3 \mathrm{fm}^{-1}$ and data are for ${ }^{56} \mathrm{Fe}$ taken from [8]. 


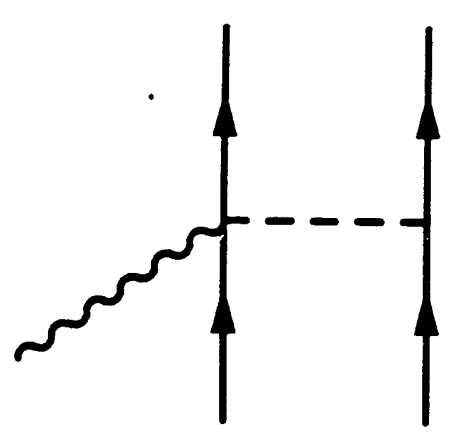

(a)

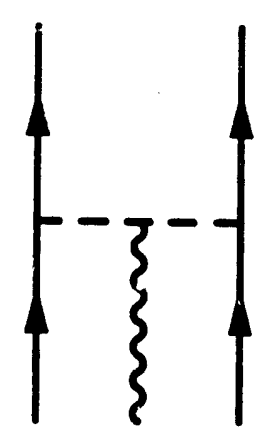

(b)

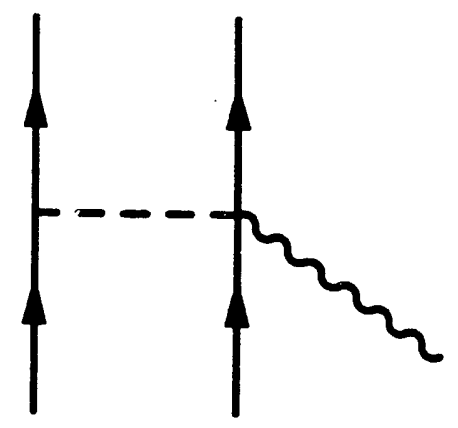

(c)

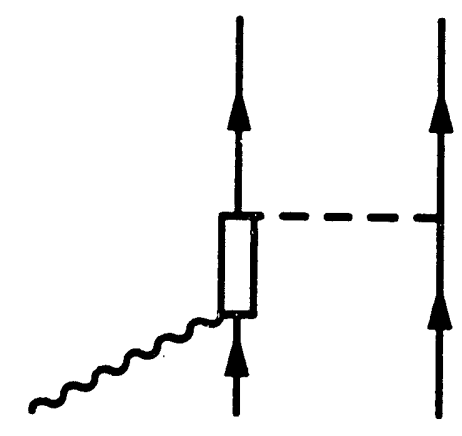

(d)

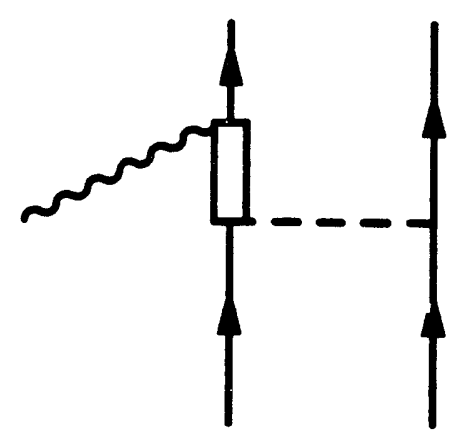

(f)

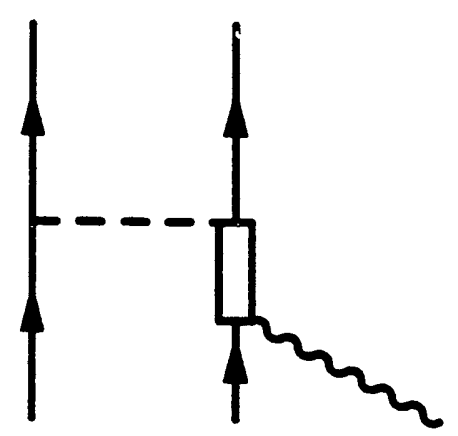

(e)

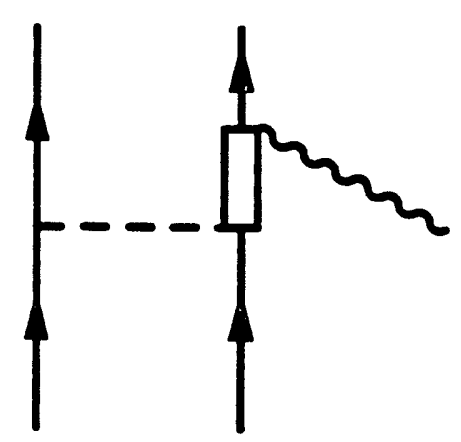

(g) 


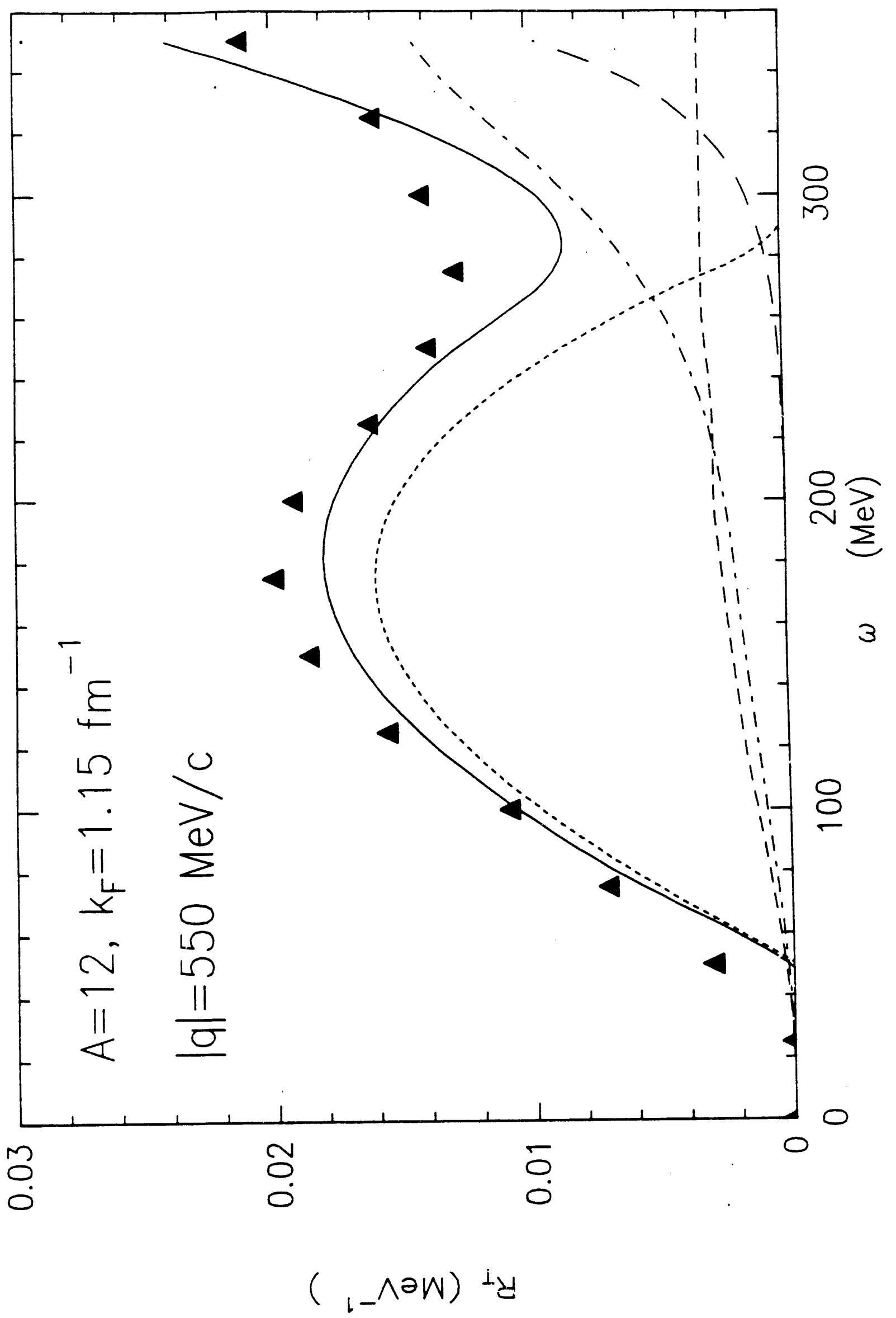



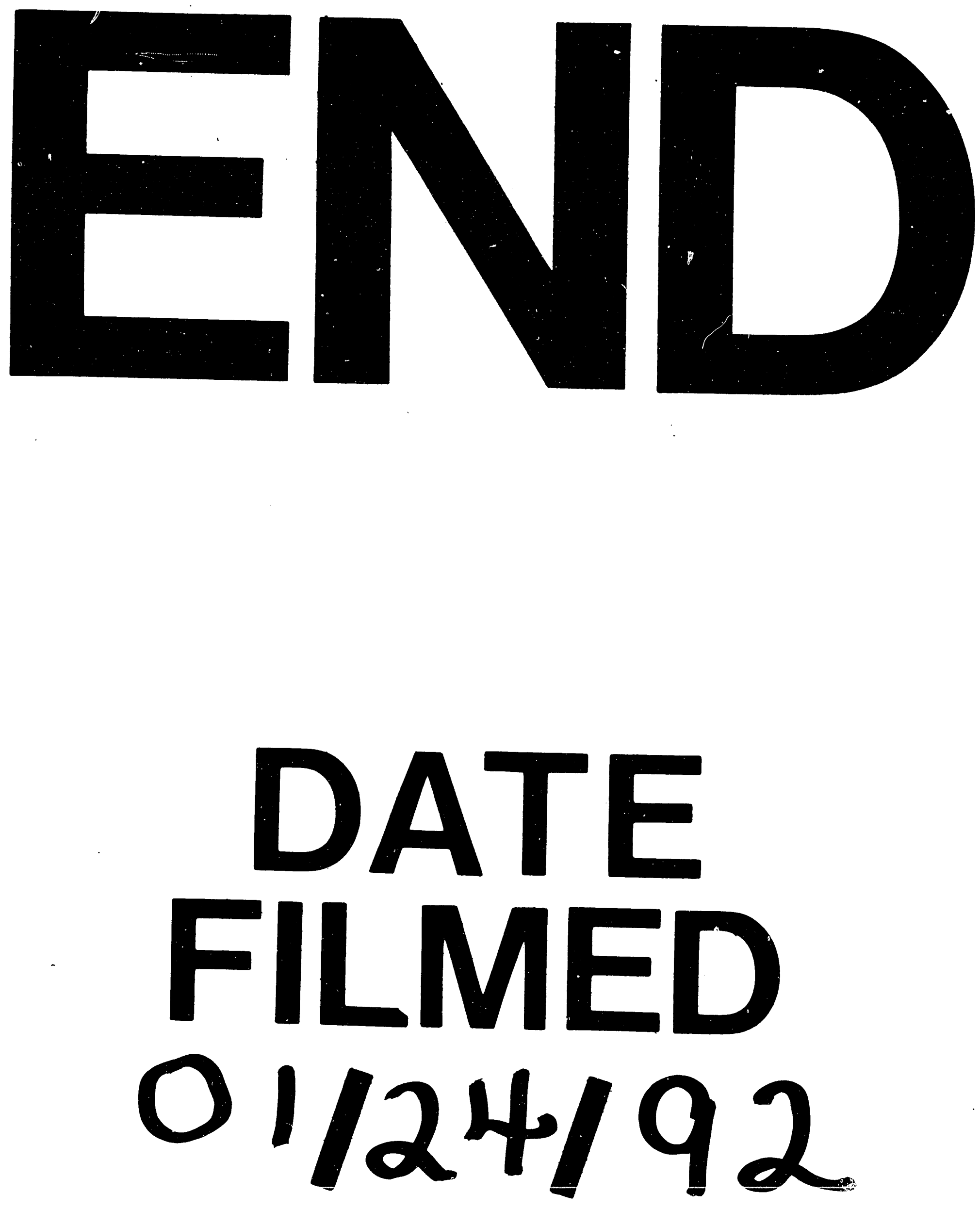


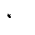

